\title{
Dosimetry Optimization System and Integrated Software (DOSIS): a comparison against Fluka code results over a standard phantom
}

\author{
Pedro Pérez ${ }^{* \dagger}$ \\ Institute of Physics E. Gaviola - CONICET \& LIIFAMIRX - Laboratorio de Investigación e \\ Instrumentación en Física Aplicada a la Medicina e Imágenes por Rayos X - University of \\ Córdoba \& Department of Physics - FCF-QyN - University of Río IV; Argentina. \\ E-mail: pperezlefamaf.unc.edu.ar
}

Francesca Botta

European Institute of Oncology, Milan; Italy

\section{Marta Cremonesi}

European Institute of Oncology, Milan; Italy

\section{Mahila Ferrari}

European Institute of Oncology, Milan; Italy

\section{Francesco Guerriero}

European Institute of Oncology, Milan; Italy

\section{Francisco Malano}

Institute of Physics E. Gaviola - CONICET \& LIIFAMIRX - Laboratorio de Investigación e Instrumentación en Física Aplicada a la Medicina e Imágenes por Rayos X - University of Córdoba; Argentina.

\section{Guido Pedroli}

European Institute of Oncology, Milan; Italy

\section{Ignacio Scarinci}

LIIFAMIRX - Laboratorio de Investigación e Instrumentación en Física Aplicada a la Medicina e Imágenes por Rayos X - University of Córdoba; Argentina

\section{Mauro Valente}

Institute of Physics E. Gaviola - CONICET \& LIIFAMIRX - Laboratorio de Investigación e Instrumentación en Física Aplicada a la Medicina e Imágenes por Rayos X - University of Córdoba; Argentina 
Actually, dual-imaging facilities allow obtainance of both mass and activity patient-specific distributions perfectly correlated, which are important to improve dose distributions estimations and radioimmunotherapy treatment planifications accuracy.

Calculus methods at voxel level require both quantitative and qualitative validation to obtain improvements in patient-specific dosimetry. The present work presents advances on the development of a novel computational tool dedicated to 3D patient-specific dosimetry at voxel level; and its results analysis and visualization. With the aim of providing a dosimetric tool for planar and tridimensional methods at voxel level, as well as the development of a platform based on fullstochastic methods for $\alpha$-, $\beta$ - and $\gamma$-emitters used in radiopharmaceutical applications. DOSIS is based on the Boltzmann radiation transport equation to realize energy delivering calculations. Procedures for 2D and 3D dosimetry have been designed tacking into accont established formalism and standards on MIRD Pamphlets. Anatomic and metabolic images, and dose maps resulting of this calculus are analysed and procesed by a special developed and designed software.

DOSIS has been preliminary validated on some standard clinic cases in comparison whith other standard procedures used commonly in radionuclide treatments, showing great accordance on its results and a friendly user usability. Finally, a dose calculation over a standard phantom is performed using DOSIS calculation code and FLUKA, validating the radiation transport code of DOSIS.

$X$ Latin American Symposium on Nuclear Physics and Applications (X LASNPA),

1-6 December 2013

Montevideo, Uruguay

\footnotetext{
${ }^{*}$ Speaker.

${ }^{\dagger}$ www.famaf.unc.edu.ar/ pperez1
} 


\section{INTRODUCTION}

Nowadays, dual-imaging techniques allow both anatomic and metabolic correlated information assessment. Facilities like PET-CT or SPECT-CT implement these techniques to obtain the corresponding information at voxel level with a millimeter resolution. Thereby, this information provides patient-specific mass and activity 3D voxelized distributions useful for improving dose distributions estimations and radioimmunotherapy treatment planifications accuracy [1].

Actually, there are several Monte Carlo codes dedicated to radiation transport and energy delivery at voxel level, both at nuclear medicine common energies involved and usual facilities resolution; like PENELOPE, Fluka, MCNP or Geant4.

Any calculation method requires both quantitative and qualitative validation, specially if it is aimed to obtain improvements in patient-specific dosimetry field [2] for nuclear medicine applications. Advances on the development of a novel computational tool dedicated to 3D patient-specific dosimetry calculation at voxel level are presented. Its results, analyses and visualization modules are shown from a general point of view, with the aim of providing an scheme of the dosimetric tool for planar and tridimensional method at voxel level and a development of a platform based on stochastic methods for $\alpha$-, $\beta$ - and $\gamma$-emitters used in radiopharmaceutical applications.

DOSIS, as it is named the presented dosimetry system, is based on the Boltzmann radiation transport equation to realize energy delivering calculations. 2D and 3D dosimetry procedures have been designed in according to the established formalism and standards on MIRD Pamphlets 16 and 17 [3]. Anatomic and metabolic images, and dose maps resulting of this calculus are analysed and procesed by a special developed and designed software [4].

DOSIS has been preliminary validated on some standard clinic cases in comparison whith other standard procedures used commonly in radionuclide treatments, showing great accordance on its results and a friendly user usability. Finally, a dose calculation over a standard phantom is performed using DOSIS calculation code and FLUKA, validating the radiation transport code of DOSIS.

\section{MATERIALS AND METHODS}

\subsection{DOSIS}

DOSIS is a novel developed dosimetry system [5] consisting mainly on complementary modules for 3D and 2D dose distribution assessment. The proposed method uses a in-home developed version of the geometry and radiation transport controllers of physic's package used by PENELOPE Monte Carlo (MC) code writen in FORTRAN 77 computational language. Also, it has a friendly graphical user interface (GUI) designed and developed on a Matlab environment which is capable of constructing 3D patient-specific activity and mass distribution from SPECT/PET and CT images, respectively, correctly previously correlated.

A general scheme of DOSIS system operation can be observe in Figure 1 and Figure 3 shows both the 3D voxel level dosimetry and planar dosimetry schemes. Further, Figure ?? presents main GUI developed for 3D and planar calculations. 


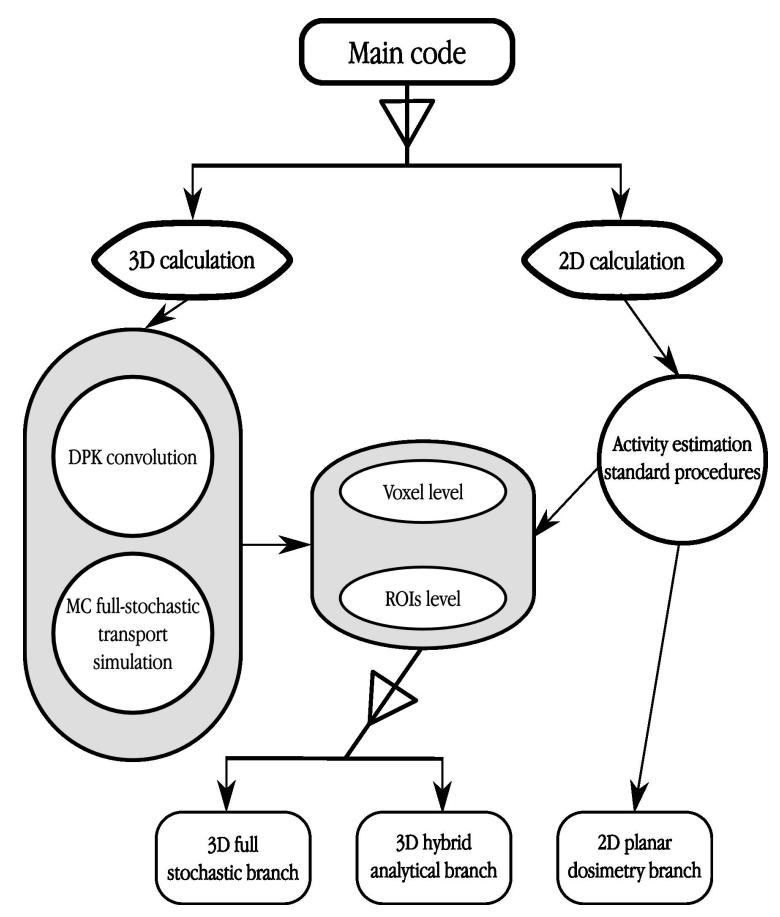

Figure 1: General schem of DOSIS dosimetry system

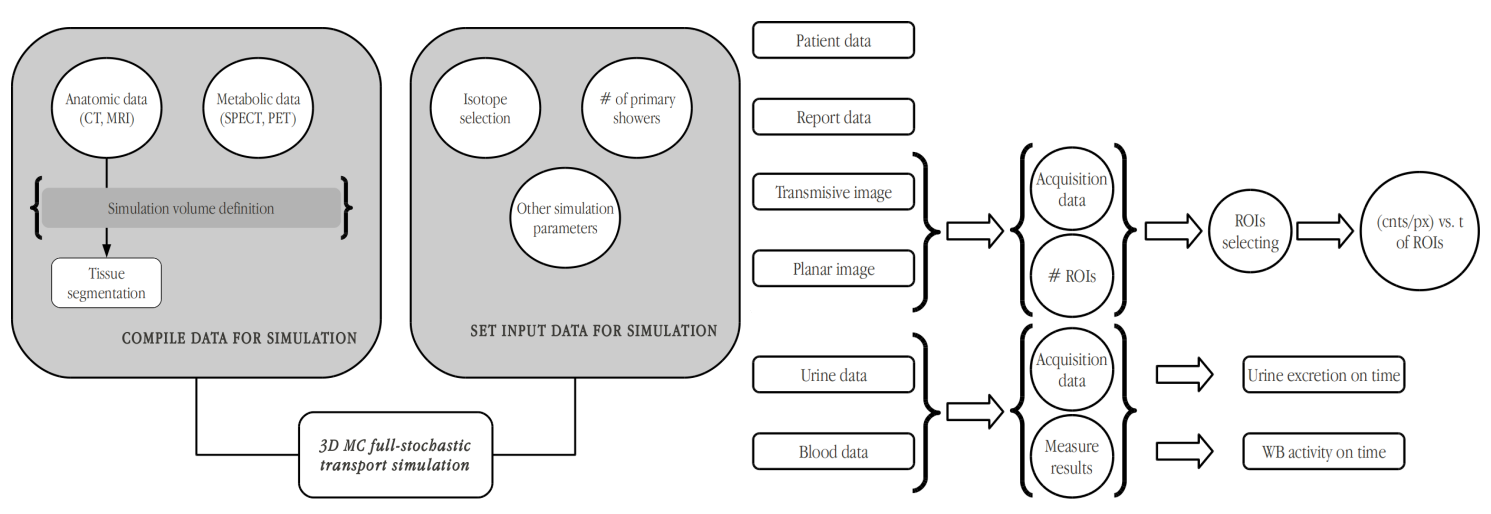

Figure 2: 3D MC calculation (left) and planar dosimetry automatization (right) schemes

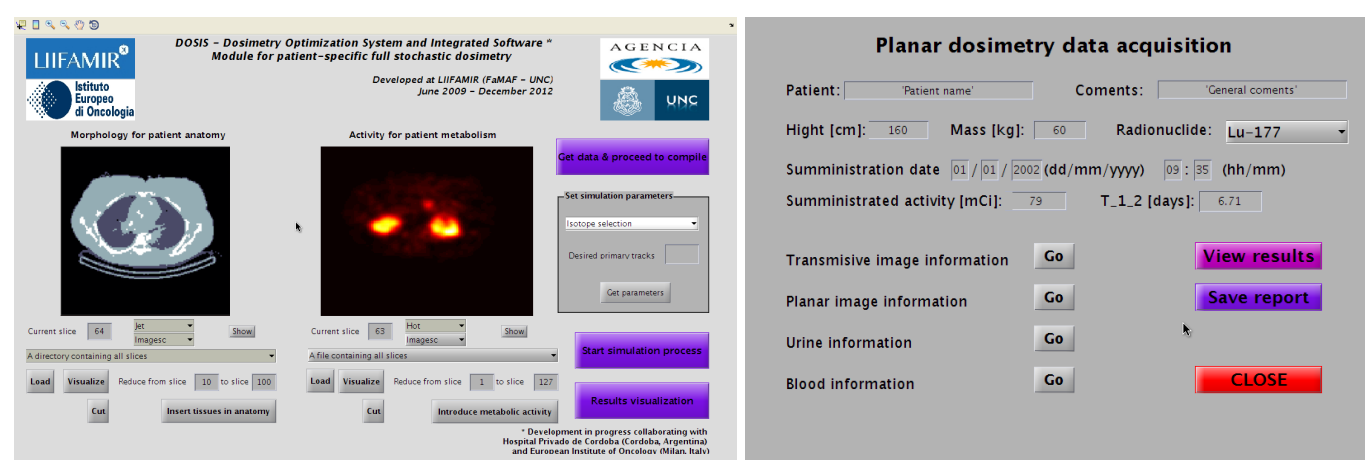

Figure 3: 3D MC calculation (left) and planar dosimetry automatization (right) main GUIs 


\subsubsection{PENELOPE MC code}

The PENetration and Energy LOss of Positrons and Electrons (PENELOPE) code [6] was partilly used for particle transport simulation and energy delivering in DOSIS. This powerful code allows photons, electrons and positrons MC.

In PENELOPE, photons tracks are generated using a detailed simulation method taking into account the succesion of events simulated in a cronological order; while electrons and positrons paths, are simulated using a mixed procedure which is very useful for energies involved in nuclear medicine procedures where a detailed simulation would have a extremely high and unnecesary computational cost.

PENELOPE is writen as a rutines serie in FORTRAN 77 language and there were specially developed a serie of packages for DOSIS capable of controlling energy deliverging and radiation transport using PENELOPE's physics packages. These packages allow to manage correctly the DOSIS constructed voxelized patient-specific mass and activity distributions.

\subsubsection{Matlab platform}

Matlab $^{1}$ is a high level language and an interactive developing environment dedicated to computation and visualization, as well as numerical programming.

This toolkit provides tools capable of realizing complex data analysis, algorithm developing and design models and applications for different scientific and technique areas. For DOSIS there were developed routines devoted to interpreting DICOM images from SPECT-CT facilities, to user-defined segmentation mass distribution of anatomic images, handle the radiation transport and energy delivering routines, dose distribution calculation and analysis of it results. Also, all the DOSIS system is operated through a GUI specially developed on Matlab environment.

\subsection{Fluka MC code}

"FLUKA is a fully integrated particle physics Monte Carlo simulation package. It has many applications in high energy experimental physics and engineering, shielding, detector and telescope design, cosmic ray studies, dosimetry, medical physics and radio-biology." 2

This code is well widespread used actually with nuclear medicine purposes [7]. For the present job, it was used to 3D voxelized dose calculation over a standard phantom with the aim of analyse DOSIS results over a similar situation.

\subsection{Standard phantoms}

There were studied standard phantoms, the first one considering an homogeneous water mass distribution and the second one taking into account inhomogeneities of bone and air inside the whole water phantom. In the first case there were inserted ${ }^{90} \mathrm{Y}$ radionuclide on a non-homogeneous distribution, whereas in the non-homogeneous phantom it was inserted the same radionuclide homogeneously over a definite region as it can be seen on images of results section.

Images were obtained by a PET-CT facility and the whole phantoms were of $200 \mathrm{~mm}$ both for its height and diameter.

\footnotetext{
${ }^{1}$ MATLAB ${ }^{\mathbb{R}}$, Primer. The MathWorks, Inc. (C) COPYRIGHT 19842013

${ }^{2} \mathrm{http} / / / \mathrm{www}$. fluka.org
} 

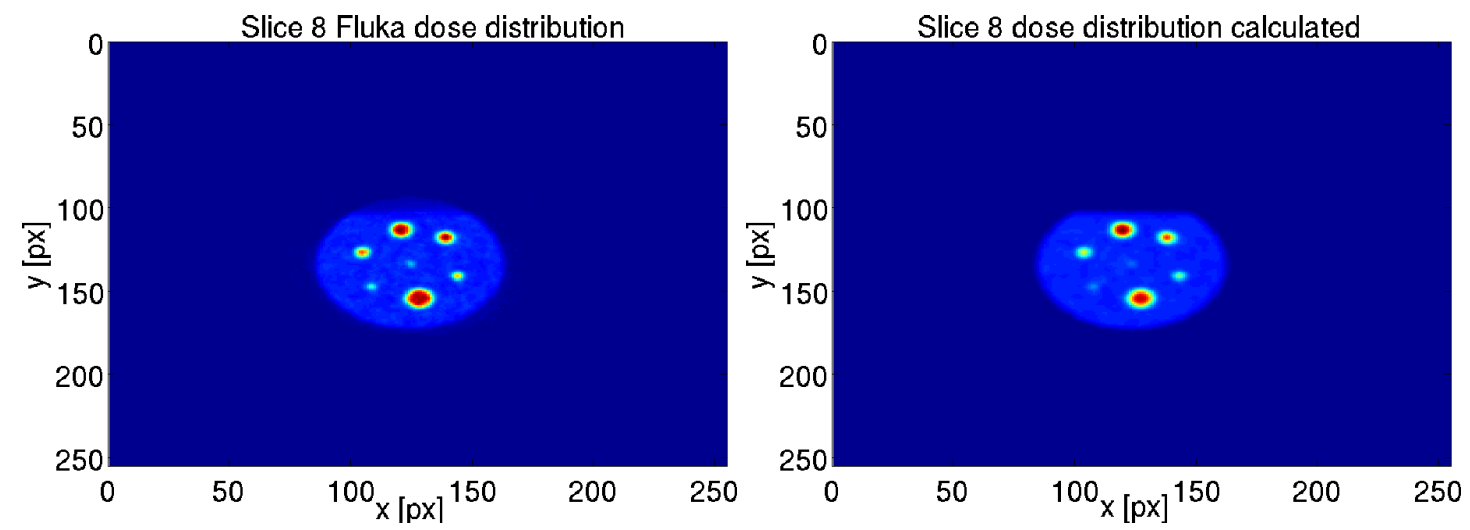

Figure 4: Dose map calculated on homogeneos phantom using Fluka (left) and DOSIS (right)
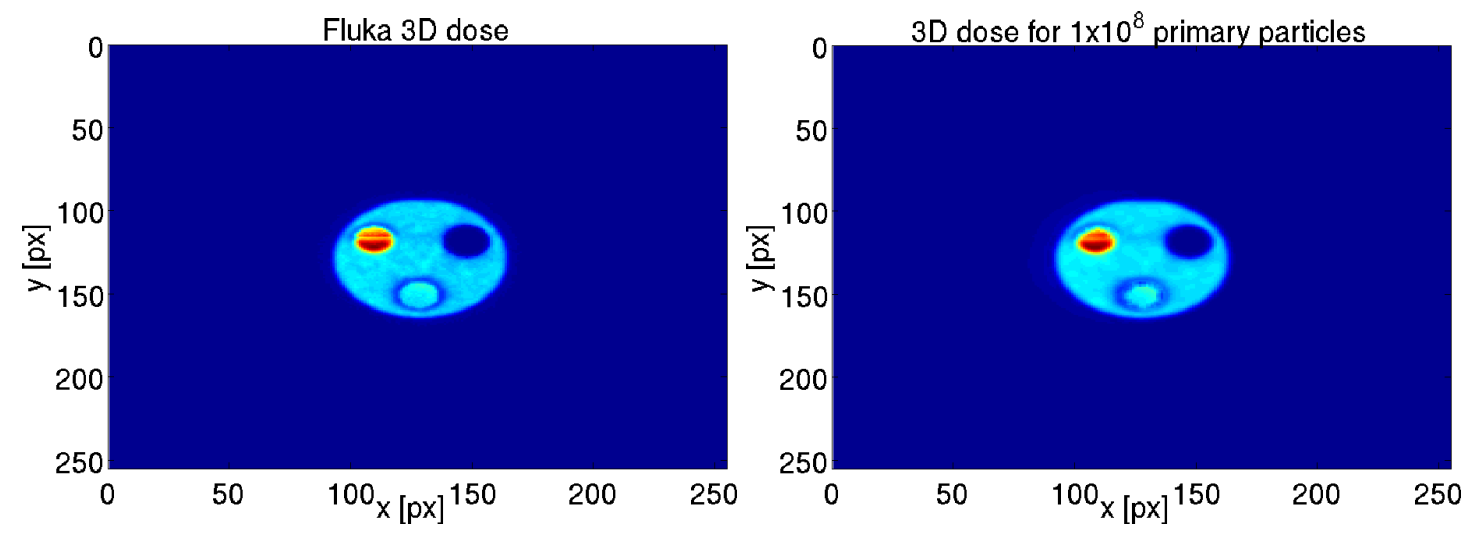

Figure 5: Dose map calculated on non-homogeneos phantom using Fluka (left) and DOSIS (right)

\subsubsection{Dose calculation}

For dose assessment by DOSIS it was considered a $10^{8}$ particles primary shower simulation and Fluka results were obtained on similar conditions.

\section{RESULTS}

Figure 4 reports dose distributions for an arbitrary slice of the water-equivalent homogeneous phantom calculated with Fluka and DOSIS, respectively. Similar results were obtained for nonhomogeneous case and it is shown in Figure 5. Exhaustive profiles comparisons were performed, as shown in Figure 6. Good agreements were obtained in all cases.

Further investigations are still in progress aimed to improve the developed tool as well as to check its performance when considering more clinical situations.

\section{CONCLUSIONS AND DISCUSSIONS}

DOSIS is a friendly toolkit offering a wide variety of dosimetry calculation methodologies and it was shown that it is possible to asses both planar dosimetry procedure calculations and voxel level dosimetry estimations. 

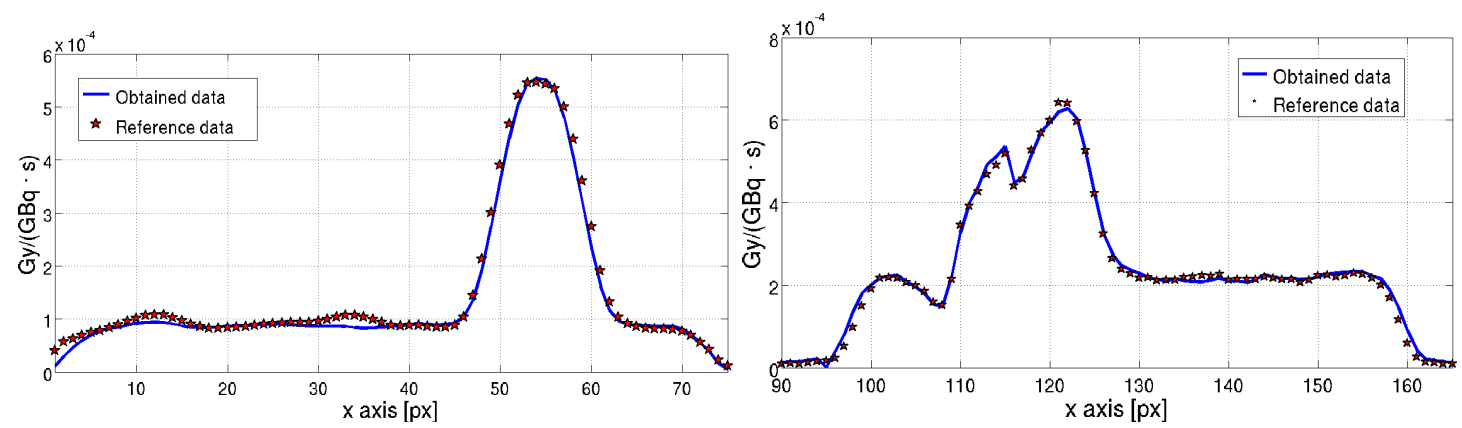

Figure 6: Dose map calculated on non-homogeneos phantom using Fluka (left) and DOSIS (right)

A preliminary comparison against other MC code in similar conditions shows good agreement in dose distribution results.

Also, further investigations over rutinely clinical situations will be done to improve the performance as a clinical application

\section{References}

[1] Y. Berker et al, Activity quantification combining conjugate-view planar scintigraphies and SPECT/CT data for patient-specific 3-D dosimetry in radionuclide therapy, Eur J Nucl Med Mol Imaging, 2011, 38(12):2173-2185.

[2] G. Sgouros, Dosimetry of Internal Emitters, J Nucl Med, 2005; 46(1):18S-27S.

[3] W.E. Bolch et al, MIRD Pamphlet No. 17: The Dosimetry of Nonuniform Activity Distributions-Radionuclide S Values at the Voxel Level, J Nucl Med, 1999; 40:11S-36S.

[4] P. Pérez et al, Dosimetry for Beta-Emitter Radionuclides by Means of Monte Carlo Simulations. 12 Chapter on Nuclear Medicine, Intech, 2010.

[5] P. Pérez et al, DOSIS (Dosimetry Optimization System and Integrated Software): An integrated toolkit for patient specific internal dosimetry, J Nucl Med, 2013; 54 (Supplement 2):1030.

[6] F. Salvat et al, PENELOPE-2008: A code system for Monte Carlo simulation of electron and photon transport, OECD Nuclear Energy Agency, Issy-les-Moulineaux, France, 2008.

[7] F. Botta et al, Calculation of electron and isotopes dose point kernels with FLUKA Monte Carlo code for dosimetry in nuclear medicine therapy, Med. Phys. 2011 Jul;38(7):3944-54. 